\title{
Practice-changing publications: Update in the management of urolithaisis
}

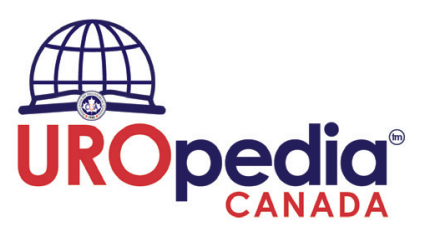

\author{
Andrea G. Lantz Powers, $M D^{\prime}$; Ben H. Chew, $M D^{2}$ \\ 'Department of Urology, Dalhousie University, Halifax, NS, Canada; '2Department of Urologic Sciences, University of British Columbia, Vancouver, BC, Canada
}

Cite as: Lantz Powers AG, Chew BH. Practice-changing publications: Update in the management of urolithaisis. Can Urol Assoc J 2021;15(8):227-9 http://dx.doi.org/10.5489/cuaj.7486

The CUA has introduced a monthly webinar series called Practice-Changing Publications (PCP series), which features Canadian experts discussing recent data that have impacted their field. The CUAJ is proud to present summaries of these talks and the important research discussed in this series.

If you missed any of the webinars, you can access them at cua.org/UROpedia and earn all-important CPD Section 3 credits!

\section{Introduction}

This article aims to highlight various areas of endourology research that have had a direct effect on clinical practice in recent years.

\section{Laser safety and technology}

Perhaps inarguably, one of the biggest changes in endourology the last number of years has been the introduction of innovative laser technology into clinical practice. For this reason, we focus on laser technology and safety as the most important update in the management of urolithiasis in recent years.

Dr. Sero Andonian led a Canadian, prospective, randomized clinical trial comparing regular and Moses (Lumenis) modes of holmium laser lithotripsy. ${ }^{1}$ In their paper, Ibrahim et al investigated the performance of the Moses mode vs. regular mode of the Lumenis 120W laser generator (LP120H Moses) to assess stone retropulsion, stone fragmentation/pulverization time, and procedural time during retrograde ureteroscopy. The Moses technology divides the holmium laser pulse into two adjacent peaks. The first peak separates the fluid ahead of the stone, creating a vapor bubble, hence the term Mosses effect, which allows the second peak to be precisely delivered to the stone with less energy loss. Laser energy transmission is less dependent on fibre-to-stone distance with the Moses mode. In their study of 72 cases, Dr. Andonian and colleagues found that the Moses mode was associated with significantly shorter fragmentation/pulverization time (14.2 minutes vs. 21.1 minutes, $p=0.03$ ) and procedural time (41.1 minutes vs. 50.9 minutes, $p=0.03$ ) compared to regular (short-pulse) mode. There was less stone retropulsion with the Moses mode (mean grade 0.5 vs. 1.0, $p=0.01$ ), which is an important consideration when treating ureteral stones. There was no difference between total energy used (10.8 kJ vs. $11.1 \mathrm{~kJ}$, $\mathrm{p}>0.05)$, treatment success rates (88.4\% vs. 83.3\%, $\mathrm{p}>0.05)$, or complications (8.3\% vs. $11.1 \%, \mathrm{p}>0.05)$.

There have been several studies that assess the thulium fibre laser (TFL) use for stones and tissue uses. The TFL has changed our practices in that the technique of stone ablation has changed from less painting to more "pop-dusting." Anecdotally, we feel that junior residents who are more novice ureteroscopists may be faster at learning laser lithotripsy, as they do not need to learn all the tips and tricks on how to perform fine movements with the ureteroscope to "paint" the face of the stone for fine dusting. Furthermore, we are expanding our clinical indications of ureteroscopy to treat larger or more complex stones less invasively rather than perform percutaneous nephrolithotomy. Other benefits are that the operating room is more pleasant, as the machine is quieter than the typical holmium laser, and the unit is very versatile, as it can be used with any standard electrical outlet. One caveat is to be aware of using high power in the ureter. Just as you would use no more than 10W cautiously with Holmium:yttrium-aluminum-garnet (Ho:YAG) in the ureter, one should not go above this level in the ureter using TFL. Similar wattage (power) from any laser equates to equal temperature rises and TFL is no different. Using higher power than 10W can result in higher temperatures that can lead to tissue ablation and stricture formation. ${ }^{2}$ Clinical use of the TFL has shown promising initial results and head-to-head trials will be likely be forthcoming in the next few years. ${ }^{3}$

Safety of the Ho:YAG laser has been assessed and a recent CUA best practice report (BPR) has been published by Bhojani et al. ${ }^{4}$ A study from 2015 using pig eyes showed that the Ho:YAG laser can produce eye injuries in the form of corneal abrasions. ${ }^{5}$ Researchers found that with a 272 micron laser fibre, the fibre needed to be within $5 \mathrm{~cm}$ of the pig's eye in order to cause damage. No retinal or lens damage 
was observed, only corneal damage. Regular prescription eyeglasses provided equivalent protection when compared to laser safety goggles. ${ }^{5}$ Accordingly, after reviewing this paper and other literature regarding the safety of Ho:YAG laser, the authors of the CUA BPR on Ho:YAG laser eye safety concluded that current evidence does not support mandatory safety eyewear for all operating room personnel and that standard eyeglasses are as protective as laser safety goggles. ${ }^{4}$

\section{Analgesia: Minimizing opioid use}

An Enhanced Recovery After Surgery (ERAS) protocol was developed by Gridley et $\mathrm{al}^{6}$ for patients undergoing ureteroscopy with a goal to minimize perioperative opioid use and have an opioid-free protocol for postoperative pain management after discharge from hospital. The ERAS protocol is summarized in Table 1. The protocol included preoperative analgesia, intraoperative use of medications to help prevent bladder pain (belladonna and opium suppositories) and renal pain (ketorolac), a tiered approach to pain relief in the postanesthesia care unit, and included no opioid prescription at discharge. Patients were discharged on acetaminophen, ibuprofen, an anticholinergic, and an alpha-blocker. Although this was not a randomized controlled trial, investigators found a significant difference pre-ERAS compared to the use of the ERAS protocol. Specifically, the mean total morphine milligram equivalent (MME) decreased from 60.1 MME to 7.7 MME $(p<0.01)$ and the percentage of patients discharged with an opioid prescription decreased dramatically from $93 \%$ to $0 \%(p<0.01)$. There was no difference in calls for postoperative pain, nor were there differences in unplanned medical encounters due to uncontrolled pain. In this study, only $28.8 \%$ of patients in the ERAS group received opioids in the recovery room. There were no differences in quality-of-life outcomes between the two groups. Although this ERAS study has some limitations (for example, the use of tramadol), it is thought-provoking and could be used to develop similar opioid reduction strategies for endourological pain management.

\section{Alpha-blockers - Beyond medical expulsive therapy}

The off-label use of preoperatively administered alpha-blockers to facilitate ureteroscopy for ureteral stones has been investigated. Pace and colleagues conducted a systematic review and meta-analysis of randomized controlled trials assessing alpha-blocker in this patient population. ${ }^{7}$ Alsaikhan et al pooled 11 randomized controlled trials comprising 1352 patients. ${ }^{7}$ They found there was a decreased need for ureteral dilation, with a $61 \%$ risk reduction (relative risk [RR] $0.39,95 \%$ confidence interval $[\mathrm{Cl}] 0.31-0.48, \mathrm{p}<0.00001)$, increased ability to reach the stone(s) (RR $1.16,95 \% \mathrm{Cl} 1.10-$ 1.23, $\mathrm{p}<0.00001$ ), improved stone-free rate (RR $1.17,95 \%$ Cl 1.08-1.26) at four-week followup, shorter operative time (weighted mean difference $-6.05,95 \% \mathrm{Cl}-10.17$ to -1.93 min, $p=0.004$ ), and shorter hospital length of stay (mean difference [MD] $-0.34,95 \% \mathrm{Cl}-0.55$ to -0.13 days, $\mathrm{p}=0.001$ ).

The meta-analysis does leave some unanswered questions and the authors note that a large, adequately powered randomized controlled trial is recommended. ${ }^{7}$ Questions remaining include: What is the optimal duration of use? What is the effect of alpha-blockers prior to flexible ureteroscopy? Do alpha-blockers facilitate ureteroscopy in the pediatric population? The meta-analysis could also not control for size of the ureteroscope due to lack of data reported. Hopefully, these questions will be answered in future studies, although studying an off-label use of a medication does present some logistical challenges.

\section{Ureteric stents}

As urologists, we often hear complaints from patients regarding symptoms related to ureteric stents. Although we sympathize with patients, many urologists may recommend ureteric stenting following ureteroscopy in the hopes of minimizing complications, such as strictures and return visits due to postoperative pain and hydronephrosis. A Cochrane review was recently published assessing the data to date regarding use of ureteral stents following uncomplicated ureteroscopy. ${ }^{8}$ This meta-analysis included 23 randomized controlled trials and 2275 patients, which represents the largest, most rigorously conducted analysis of this data to date. Unfortunately, the authors were unable to conduct pre-planned subgroup analysis of clinical data due to lack of more granular reporting in each study and there may have been selective reporting bias, as none of the 23 studies had been prospectively registered. The low-quality data

\begin{tabular}{|c|c|}
\hline \multirow[t]{2}{*}{ Preoperatively } & Acetaminophen $650-1000 \mathrm{mg} \mathrm{PO}$ \\
\hline & Gabapentin $100-300$ mg PO \\
\hline \multirow[t]{2}{*}{ Intraoperatively } & Belladonna/opium 30 mg PR \\
\hline & Ketorolac 300 mg IV \\
\hline \multirow[t]{3}{*}{$\begin{array}{l}\text { Post-anesthesia } \\
\text { care unit }\end{array}$} & $\begin{array}{c}\text { First-line: Tramadol } 50 \mathrm{mg} \text { PO as needed for } \\
\text { pain rated } 4-7 / 10\end{array}$ \\
\hline & $\begin{array}{c}\text { Second-line: Oxycodone } 5 \mathrm{mg} \text { PO as needed for } \\
\text { pain rated } 4-7 / 10\end{array}$ \\
\hline & Hydromorphone $0.25 \mathrm{mg}$ IV for pain rated $\geq 8 / 10$ \\
\hline \multirow[t]{4}{*}{ Discharge } & $\begin{array}{l}\text { Acetaminophen } 1000 \text { mg every } 8 \text { hours for } \\
7 \text { days }\end{array}$ \\
\hline & Ibuprofen $800 \mathrm{mg}$ every 8 hours for 7 days \\
\hline & Anticholinergic $10 \mathrm{mg} \mathrm{XL}$ daily prn for 10 days \\
\hline & Alpha-blocker $0.4 \mathrm{mg}$ daily for 10 days \\
\hline
\end{tabular}


resulted in limited findings in the meta-analysis. Some of the potential benefits of ureteric stenting were reported to be fewer unplanned emergency room visits, less narcotic use, fewer urinary tract infections, fewer strictures, and fewer unplanned hospitalizations. The certainty of evidence was moderate to very low for all these findings and none reach statistical significance (Table 2). A downside of stenting was the possible finding of more secondary interventions but the certainty of evidence for this finding was low. Higher-quality data is still needed to definitively answer the question of the benefit of routine stenting for uncomplicated ureteroscopy.

Using a stent tether has been shown to have no difference in pain scores or urinary symptoms in the postoperative period. Stents with strings generally are left for shorter indwelling time and there is up to $10 \%$ dislodgement with strings. ${ }^{9,10}$ Additional benefits of using stent tethers is that they can be removed at home, resulting in less financial costs to the healthcare system and patients, as well as increased convenience for patients, and they are associated with less patient morbidity. Patients report less discomfort and lower visual analog scale scores using a string tether for removal when compared to cystoscopic removal. ${ }^{9,10}$

Length of time for stenting is controversial and varies by provider. In a 2017 study, Paul et al compared length of stenting of three days vs. seven days. ${ }^{11}$ They found that the post-procedure-related events, such as phone calls, unplanned clinic visits, and emergency room visits, were higher in the three-day stent group at $39 \%$ vs. $21 \%$ in the seven-day stent group. Overall, patients in the seven-day group did better than those in the three-day group. Studies from the Lange laboratory show that at three days, ureteral peristalsis decreases and this loss may result in less pain at seven days. ${ }^{12}$ Removing a stent after only three days may result in more ureteral spasm and thus, more patient pain.

Kim et al performed a recent study comparing $5 \mathrm{~F}$ to $6 \mathrm{~F}$ ureteral stents and looked at quality of life (QOL) and patient-reported outcome measures (PROM). ${ }^{13}$ They found no difference in QOL or PROM scores between these two different stent sizes.

The infunidbulo-pelvic angle (IPA) has traditionally been a factor in our decision of which modality to use to treat lower pole stones. In a study looking at stone-free rate and IPA angles, IPA $<90$ degrees portended a lower stone-free rate. ${ }^{14}$ IPA $<90$ degrees equates to a very acute angle into the lower pole, making it more difficult to access ureteroscopically. Consideration should be given to mini or standard percutaneous nephrolithotomy in these cases. Moving the stone into an upper pole calyx, when possible, also increases the stone-free rate.

\section{Conclusions}

These were some of the more practice-changing papers we found in the literature over the last two years. It is great to see our field taking both major and minor steps to help us treat our patients more effectively.

Competing interests: Dr. Chew has been an advisory board member for ADVA-Tec, Ambu, Auris Surgical, Becton Dickinson, Boston Scientific, Cook Medical, Olympus, Storz Medical, Sonomotion, and The Ureteral Stent Company; has received grants/honoraria from ADVA-Tec, Ambu, Auris Surgical, Becton Dickinson, Boston Scientific, Cook Medical, Olympus, Storz Medical, Sonomotion, and The Ureteral Stent Company; hold investments in Auris Surgical, Sonomotion, and The Ureteral Stent Company; and has participated in clinical trials supported by ADVA-Tec, Boston Scientific, Cook Medical, Olympus, Storz Medical, and Sonomotion. Dr. Lantz Powers reports no competing personal or financial interests related to this work.

\section{References}

1. Ibrahim A, Ehilali MM, Fahmy N, et al. Double-blinded, prospective, randomized clinical trial comparing regular and Moses modes of holmium laser lithotripsy. J Endourol 2020;34:624-8. https://doi. org/10.1089/end.2019.0695

2. Molina WR, Carrera RV, Chew BH, et al. Temperature rise during ureteral laser lithotripsy: Comparison of super pulse thulium fiber laser (SPTF) vs. high power 120 W holmium-YAG laser (Ho:YAG). World I Urol 2021. [Epub ahead of print]. https://doi.org/10.1007/s00345-021-03619-3

3. Carrera RV, Randall JH, Garcia-Gil M, et al. Ureteroscopic performance of high-power super pulse thulium fiber laser for the treatment of urolithiasis: Results of the first case series in North America. Urology 2021. [Epub ahead of print]. htrps://doi.org/10.1016/i.urology.2020.12.054

4. Bhojani N, Andonian S, Watterson JD, et al. Canadian Urological Association best practice report: Holmium:YAG laser eye safety. Can Urol Assoc J 2020;14:380-2. https://doi.org/10.5489/cuaj.6941

5. Villa L, Cloutier J, Comperat E, et al. Do we really need to wear proper eye protection when using holmium:YAG laser during endourologic procedures? Results from an ex-vivo animal model on pig eyes. J Endourol 2016;30:332-7. https://doi.org/10.1089/end.2015.0232

6. Gridley C, Robles J, Calvert J, et al. Enhanced recovery after surgery protocol for patients undergoing ureteroscopy: Prospective evaluation of an opioid-free protocol. J Endourol 2020;34:647-53. https://doi.org/10.1089/end.2019.0552

7. Alsaikhan B, Koziarz A, Lee JY, et al. Preoperative alpha-blockers for ureteroscopy for ureteral stones: A systematic review and meta-analysis of randomized controlled trials. J Endourol 2020;34:33-41. htrps://doi.org/10.1089/end.2019.0520

8. Ordonez $M$, Hwang $E C$, Borofsky $M$, et al. Ureteral stent versus no ureteral stent for ureteroscopy in the management of renal and ureteral calculi. Cochrane Database Syst Rev 2019;2:CD012703. htrps://doi.org/10.5489/cuai.5957

9. Inoue $T, 0$ akada $S$, Hamamoto $S$, et al. Impact of ureteric stent removal by string on patient's quality of life and on complications at post-ureteroscopy for urolithiasis: A controlled trial. BJU Int 2019;124:314-20. https://doi.org/10.1111/bju.14622

10. Sun $X$, Dong $L$, Chen $T$, et al. Pooled analysis of efficacy and safety of ureteral stent removal using an extraction string. Medicine (Baltimore) 2019;98:e17169. https://doi.org/10.1097/ MD.0000000000017169

11. Paul CJ, Brooks NA, Ghareeb GM, et al. Pilot study to determine optimal stent duration following ureteroscopy: Three vs. seven days. Curr Urol 2018;11:97-102. https://doi.org/10.1159/000447201

12. Huang ZA, Scotland KB, Li Y, et al. Determination of urinary prostaglandin E2 as a potential biomarker of ureteral stent associated inflammation. J Chromatogr B Analyt Technol Biomed Life Sci 2020;1145:122107. https://doi.org/10.1016/i.ichromb.2020.122107

13. Kim BS, Choi JY, Jung W. Does a ureteral stent with a smaller diameter reduce stent-related bladder irritation? A single-blind, randomized, controlled, multicenter study. J Endourol 2020;34:368-72. htrps://doi.org/10.1089/end.2019.0482

14. Dresner SL, Iremashvili V, Best SL, et al. Influence of lower pole infundibulopelvic angle on success of retrograde flexible ureteroscopy and laser lithotripsy for the treatment of renal stones. J Endourol 2020;34:655-60. https://doi.org/10.1089/end.2019.0720

Correspondence: Dr. Andrea G. Lantz Powers, Department of Urology, Dahousie University, Halifax, NS, Canada; aglantz@dal.ca 